\title{
Common variants of genes encoding TLR4 and TLR4 pathway members TIRAP and IRAK1 are effective on MCP1, IL6, IL1 $\beta$, and TNFa levels in type 2 diabetes and insulin resistance
}

\author{
Irfan Degirmenci ${ }^{1} \cdot$ Cansu Ozbayer ${ }^{2}(0) \cdot$ Medine Nur Kebapci $^{3} \cdot$ Hulyam Kurt $^{4} \cdot$ Ertugrul Colak $^{5} \cdot$ Hasan Veysi Gunes $^{4}$
}

Received: 9 May 2019 / Revised: 24 May 2019 / Accepted: 12 June 2019 / Published online: 20 June 2019

(c) Springer Nature Switzerland AG 2019

\begin{abstract}
Objective and design Type 2 diabetes is a pandemic disease characterized by hyperglycemia, ineffective insulin use, and insulin resistance and affecting 1 in 11 people worldwide. Inflammation-related insulin resistance is thought to play an important role in the etiology of the disease. TLR4 is the central receptor of the natural immune system and has an important role as a trigger of the inflammatory response. The IRAK1 and TIRAP are members of the TLR4 pathway and involved in the TLR4-mediated inflammatory response. Genetic variants in the TLR4 gene or in the IRAK1 and TIRAP genes may have an important role in the development of insulin resistance and type 2 diabetes by disrupting the inflammatory response. In this direction, we aimed to investigate the relationship among TLR4 and IRAK1, TIRAP gene variants, and type 2 diabetes and insulin resistance, and investigate how these variants affect inflammatory factors (TNF- $\alpha$, IL-6, MCP-1, and IL-1 $\beta$ ). Subjects and methods In our study, a total of seven variations on the genes of TLR4 (rs4986790, rs4986791), IRAK1 (rs1059703, rs3027898, rs7061789), and TIRAP (rs8177374, rs8177400) were genotyped by the MassARRAY ${ }^{\circledR}$ Iplex GOLD SNP genotyping in 100 type 2 diabetic patients and 100 non-diabetic individual. The TLR4 rs4986790 and rs4986791 variation was confirmed by PCR-RFLP method also. The serum IL1- $\beta$, IL6, MCP-1, and TNF- $\alpha$ levels were measured using enzyme-linked immunosorbent assay kits.

Results and conclusion As a result of our study, no correlation was found among TLR4, IRAK1, and TIRAP gene variants and the risk of type 2 diabetes and insulin resistance. However, TNF- $\alpha$, IL-6, MCP-1, and IL-1 $\beta$ levels were also associated with diabetes and insulin resistance $(p>0.05)$. Although the gene variants were not significant in type 2 diabetes and insulin resistance groups, IRAK1, TLR4, and TIRAP gene variants were found to be associated with TNF- $\alpha$, IL-6, MCP-1, and IL-1 $\beta$ levels.
\end{abstract}

Keywords Type 2 diabetes · Insulin resistance $\cdot$ Inflammation $\cdot$ TLR4 $\cdot$ IRAK $1 \cdot$ TIRAP

Responsible Editor: John Di Battista.

Cansu Ozbayer

c.ozbayer@gmail.com

1 Department of Medical Biology, Medical Faculty, Kutahya Health Sciences University, Kutahya, Turkey

2 Faculty of Health Sciences, Kutahya Health Sciences University, Kutahya, Turkey

3 Department of Endocrinology, Medical Faculty, Eskisehir Osmangazi University, Eskisehir, Turkey

4 Department of Medical Biology, Medical Faculty, Eskisehir Osmangazi University, Eskisehir, Turkey

5 Department of Biostatistics, Medical Faculty, Eskisehir Osmangazi University, Eskisehir, Turkey

\section{Introduction}

Although inflammation is an important component of the natural immune response, prolonged chronic inflammation can lead to metabolic diseases such as insulin resistance, obesity, and type 2 diabetes [1-3].

Type 2 diabetes (T2DM) is a disease characterized by hyperglycemia and is caused by complete or partial deficiency of insulin. Insulin deficiency, as well as insulin resistance (IR), plays an important role in the development of disease and carbohydrate, lipid, and protein metabolism also affects [4].

Insulin resistance is defined as a decrease in biological response to normal concentration of insulin in the circulation and is the primary characteristic of type 2 diabetes. 
Insulin resistance leads to excessive insulin secretion from the pancreatic $\beta$ cells and, thus, compensating hyperinsulinemia [5-7].

Insulin resistance is associated with many complex factors such as genetic, nutritional, and lifestyle. In addition, regulators synthesized from the immune system and adipose tissue are involved in the regulation of insulin level. Insulin binds to its specific receptor in all cells and initiates many intracellular cascades. The insulin receptor stimulates tyrosine phosphorylation. This step is also one of the key molecular steps in inflammation-related insulin resistance. Serine phosphorylation with various inflammatory signals is one of the most important causes of the degradation of the insulin receptor signal [7-10].

The most important of these inflammatory mechanisms are associated with cytokines and chemokines. Many of the proinflammatory adipokines, such as IL (interleukin)- $1 \beta$, IL-6, IL-8, tumor necrosis factor (TNF)- $\alpha$, and MCP-1 (monocyte chemoattractant protein), are expressed by white adipose tissue and all affect insulin resistance. Activation of the TLR4 signal by long-chain fatty acids and lipopolysaccharide (LPS) in adipocytes causes activation of nuclear factor kappa B (NF-kB) and expression of inflammatory regulatory genes, such as TNF- $\alpha$, IL- 1 , IL-6, and MCP-1 [8, 11]. Inflammation disrupts insulin sensitivity by activation of the TLR family, in particular TLR4 [12, 13].

Toll-like receptors (TLR) are a group of transmembrane proteins that provide the natural immune response to many pathogens. It is a homolog of the interleukin-1 receptor (IL-1R) in humans and also has an important role in host immunity by enabling the adaptive immune response to be activated $[14,15]$.

TLR4 is the central receptor of the natural immune system and is expressed in cardiomyocytes, macrophages, tracheal epithelium, and endothelial and smooth muscle cells. TLR4 plays an important role in the recognition of microbial components, especially LPS [16].

TLR4 induces a series of kinase pathways and transcription factor activation to induce a natural immune response to pathogens. In the TLR 4 signaling, two pathways, MyD88 (myeloid differentiation protein 88 ) dependent pathway and MyD88 independent pathway, were identified. The triggering of signal cascades in the MyD88-dependent pathways causes the formation of natural immunity factors such as proinflammatory cytokines, chemokines, eicosanoids, and reactive oxygen species (ROS) [12].

The activation of Myd88-mediated TLR4 signaling pathway occurs as follows; CD14 recognizes bacterial LPS, initiates signaling by forming a complex with TLR4 and MD-2. Myd88 binds to TLR4 in the presence of TIRAP and incorporates IRAK complex into the complex. Then, the IRAK is separated from the complex and TRAF is included in the complex. Herewith, the NF- $\mathrm{KB}$ and regulatory genes are activated (Fig. 1) [17].

Interleukin-1 receptor-associated kinases (IRAKs) are an important family of death sites that play an important role in signal cascades of two receptor families (TLR and IL-1R). The IRAK1, member of the IRAK family, is involved in the TLR-related inflammation mechanism and important pathway for various autoimmune and inflammatory diseases such as diabetes and atherosclerosis [18].

Adapter protein-containing TIR domain (TIRAP) provides activation of NF- $\mathrm{KB}$ and mitogen activated protein (MAP) kinases by TLR2 and TLR4-mediated MyD88dependent signaling pathway and is specific for TLR2 and TLR4 [19].

Briefly, the mechanism of inflammation is an important mechanism affecting the development of insulin resistance and, thus, type 2 diabetes. TLR 4 can affect the development of insulin resistance in type 2 diabetes through inflammatory regulators such as TNF- $\alpha$, IL-1, IL-6, and MCP-1 with the MyD88-dependent pathway. Therefore, genetic variants in the TLR4 gene or in the IRAK and TIRAP genes that are members of the MyD88-dependent TLR4 pathway may have an important role in the development of type 2 diabetes and insulin resistance.

In this direction, (1) determining the relationship between TLR4 and IRAK1, TIRAP gene variants and type 2 diabetes and insulin resistance and (2) investigating how these variants affect inflammatory factors (TNF- $\alpha$, IL-6, MCP-1, and IL-1 $\beta$ ) were aimed.

\section{Materials and methods}

\section{Patient selection}

The 100 type 2 diabetic patients and 100 non-diabetic individual that enrolled at the Department of Endocrinology, Eskisehir Osmangazi University, Medical Faculty in Turkey were included this study. The study was approved by the local ethical committee of Eskisehir Osmangazi University (Approval no: 2013/62) and informed consent forms signed all participants prior to study according to Helsinki Declaration.

The type 2 diabetic patients with insulin resistance group (T2DM IR+) consist of 75 individual, type 2 diabetic patients without insulin resistance group (T2DM IR-) consist of 25 individual, insulin resistance patients without type 2 diabetes group (IR+) consist of 22 individual, and control group consist of 78 individual.

The HbA1c, fasting glucose, triglyceride, LDL values, fundus examination, neuropathy, nephropathy, and albuminuria were considered for clinical diagnosis of type 2 diabetes. Homeostatic model assessment insulin resistance 

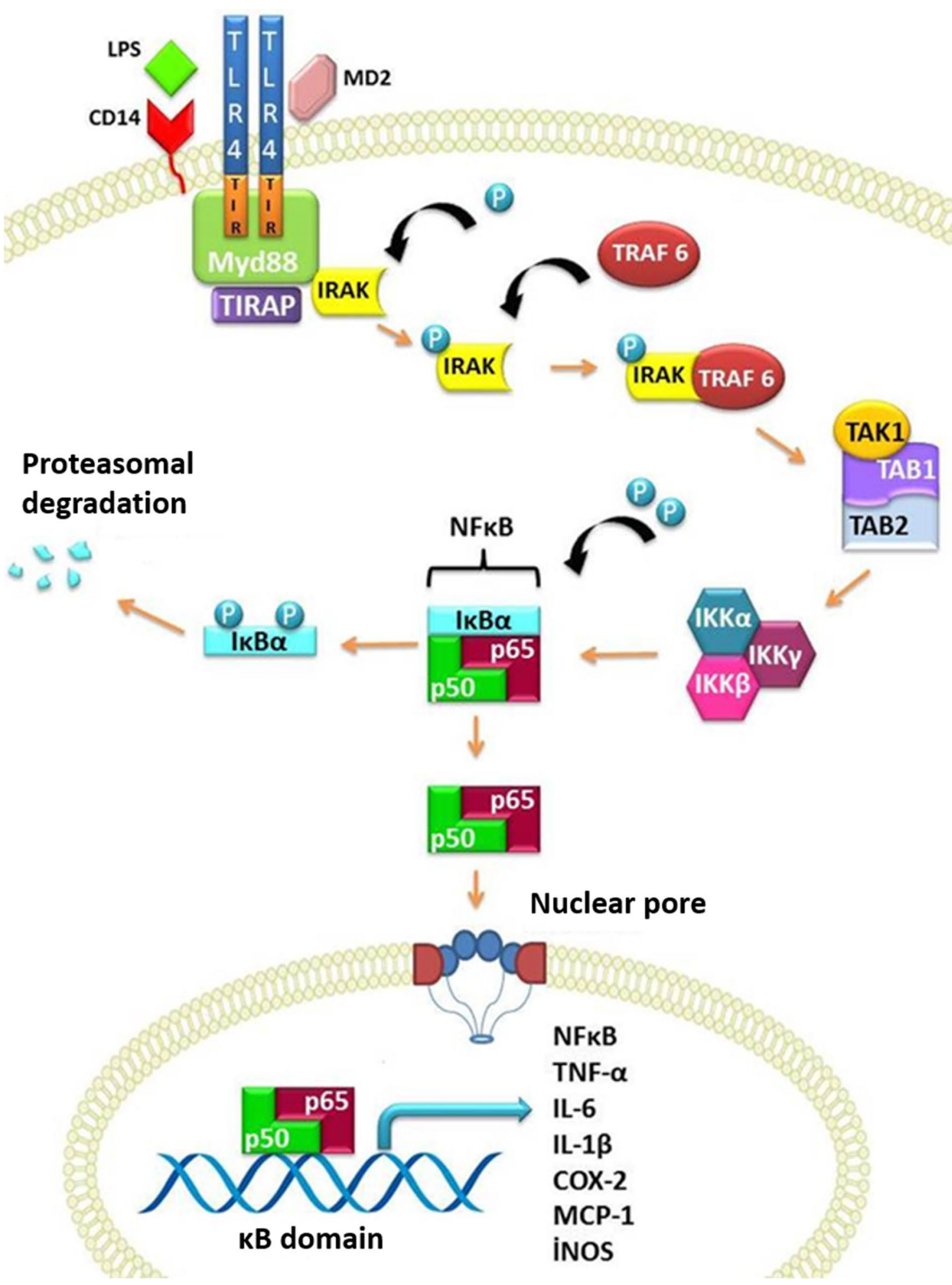

Fig. 1 Myd88-mediated TLR4 signaling pathway [15]

(HOMA-IR) scores higher than 2.7 were considered to be insulin resistant. All biochemical parameters were recorded from clinical file of patients.
Individuals under the age of 25 or older than 75 years, individuals with any chronic metabolic disease other than diabetes and insulin resistance syndrome, for control group, 
and individuals with a history of diagnosis and/or treatment of type 2 diabetes themselves or their primary relatives were not included in the study.

\section{DNA isolation and quantification}

PureLinkTM Genomic DNA Mini Kit (Invitrogen Corporation, Carlsbad, California, USA) was used for DNA isolation and all isolation steps were performed according to the kit protocol. The amount and purity of the DNA samples obtained by the DNA isolation kit were measured by Thermo Scientific NanoDrop ${ }^{\circledR} 1000$ (Thermo Fisher Scientific Inc., Wilmington, DE, USA) spectrophotometer.

\section{Genotyping}

In our study, a total of seven variations on the genes of TLR4 (rs4986790, rs4986791), IRAK1 (rs1059703, rs3027898, rs7061789), and TIRAP (rs8177374, rs8177400) were genotyped by the MassARRAY ${ }^{\circledR}$ Iplex GOLD SNP genotyping. The TLR4 rs4986790 and rs4986791 variation was confirmed by polymerase chain reaction-restriction fragment length polymorphism (PCR-RFLP) method also.

\section{MassARRAY ${ }^{\circledast}$ Iplex GOLD SNP genotyping}

The TLR4, IRAK1, and TIRAP gene variants were analyzed by the Iplex GOLD SNP genotyping protocol using the Sequenom MassARRAY ${ }^{\circledR}$ System (Sequenom Inc., San Diego, California, USA). The sequence design of amplification and elongation primers for SNPs was performed in Sequenom Assay Designer software and primers obtained from the manufacturer (Metabion, Germany).

After multiplex PCR amplification, SAP (Shrimp Alkaline Phosphatase) (Sequenom Inc.) reaction, primer elongation reaction (Iplex GOLD reaction), and resin purification, the reaction products in the PCR plate were transferred onto the SpectroCHIP with the MassARRAY TM Nanodispenser RS1000.

PCR products transferred onto SpectroCHIP were placed on the MassARRAY analyzer conducted the matrix assisted laser desorption ionization time of flight (MALDI-TOF) mass spectrometer technique. The MassARRAY analyzer reported all genotype results based on the mass analysis.

\section{PCR-RFLP verification of TLR4 rs4986790 and rs4986791 variation}

The PCR amplification reaction was prepared with DNA, PCR mix (OneTaq ${ }^{\circledR}$ Quick-Load $^{\circledR}$ 2X Master Mix with Standard Buffer, New England Biolabs, Ipswich, MA, USA), forward-reverse primers (Alpha DNA, Montreal, Canada), and $\mathrm{ddH} 2 \mathrm{O}$. The reaction was carried out in the Bio-Rad
Thermal Cycler (T100 TM, Foster City, CA, USA) according to the master mix protocol.

Primer sequences for the TLR4 rs4986790; F: 5'-AGC ATACTTAGACTA CTACCTCCATG-3' and R: 5'-GAG AGATTTGAGTTTCAATGTGGG-3', for rs4986791; F: 5'-GGTTGCTGTTCTCAAAGTGATTTTGGGAGAA-3' and R: 5'-GGA AATCCAGATGTTCTAGTTGTTCTA AGCC-3'.

The PCR products digested with $1 \mathrm{U}$ of $\mathrm{NcoI}$ (New England Biolabs, Inc., Beverly, USA) for rs4986790 and $1 \mathrm{U}$ of HinfI (New England Biolabs, Inc., Beverly, USA) with incubation at $37^{\circ} \mathrm{C}$ for $1 \mathrm{~h}$.

The digested PCR products were electrophoresed in $2 \%$ agarose gel containing RedSafe TM nucleic acid dye (Intron Biotechnology Inc., Seoul, Korea) and evaluated with GeneGenius Gel Light Imaging System (Syngene, Cambridge, UK).

\section{ELISA assay}

The serum IL1- $\beta$, IL6, MCP-1, and TNF- $\alpha$ levels were measured using enzyme-linked immunosorbent assay kits (Elabscience Biotechnology Co., Ltd., Wuhan, China) and Sandwich-ELISA method by MultiskanGO UV/VIS microplate spectrophotometer (Thermo Scientific, MA, USA).

\section{Statistical analysis}

The Shapiro-Wilk test was used to investigate the consistency of continuous data (duration, year, etc.) to normal distribution. The comparison of continuous variables with normal distribution was performed by Kruskal-Wallis analysis.

Chi-square analysis (Pearson and exact Chi-square) were used for the comparison of categorical variables such as the distribution of genotypes and alleles. In the pairwise comparison of genotypes according to the groups, two ratio tests (Fisher's exact test) were used. Odds ratios were calculated by univariate and multivariate logistic regression analyses.

Statistical analyses were performed with IBM SPSS Statistics 21 package program. Tests for deviation from Hardy-Weinberg equilibrium (HWE), and differences in dominant, recessive, homozygous and heterozygous genetic models were also performed using the FINNETI program (https://ihg.gsf.de/cgi-bin/hw/hwa1.pl). The $p$ value less than 0.05 was considered significant.

\section{Results}

\section{Demographic and biochemical findings}

The demographic characteristics of non-diabetic and T2DM individuals were evaluated and their statistical evaluations 
are presented in Table 1. Age, weight, and body mass index (BMI) averages were found to be different in non-diabetic and T2DM subjects at $p<0.001$ and height at $p=0.002$. There was no statistically significant difference between male and female gender distribution in control and T2DM subjects $(p>0.05)$.

When non-diabetic and T2DM individuals and IR(+) and IR(-) subgroups were compared in terms of glucose, insulin, HbA1C, triglyceride, LDL, and creatinine levels; glucose, insulin, $\mathrm{HbA} 1 \mathrm{C}$, and triglyceride levels were found to be significant $(p<0.001)$. The multiple comparisons of glucose, insulin, HbA1C, triglyceride, LDL, and creatinine levels were also evaluated, and their statistical evaluations are presented in Table 2.

\section{Genotype findings}

The genotype distributions of TLR4, IRAK1, and TIRAP are represented in Table 3. The TLR4 rs4986790 and rs4986791 variations showed similar genetic distribution both of non-diabetic and type 2 diabetic individual. There was no statistically significant association for these variants in IR subgroups either $(p<0.05)$. TLR4 rs4986790 and rs4986791 genotype frequencies were in Hardy-Weinberg equilibrium.

When IRAK1 rs1059703, rs3027898, and rs3027898 genotypes were compared, we did not find any correlation among these variants in non-diabetic, type 2 diabetic group, and IR subgroups ( $p<0.05)$. IRAK1 rs1059703, rs3027898, and rs3027898 genotypes were deviate from Hardy-Weinberg equilibrium, contrary to expectations.

Similarly, TIRAP rs8177374 and rs8177400 genotypes were not significant in all groups $(p<0.05)$. The rs8177374 and rs8177400 genotype frequencies were in Hardy-Weinberg equilibrium.

Table 1 Demographic data of the study population

\begin{tabular}{|c|c|c|c|c|c|c|c|c|}
\hline \multirow[t]{2}{*}{ Demographic features } & \multicolumn{3}{|c|}{ Non-diabetic (mean $\pm \mathrm{SD})$} & \multicolumn{4}{|c|}{ Type 2 diabetic $($ mean \pm SD) } & \multirow[t]{2}{*}{$p$} \\
\hline & $\mathrm{IR}+(n=22)$ & \multicolumn{2}{|c|}{$\mathrm{IR}-(n=78)$} & \multicolumn{2}{|c|}{$\mathrm{IR}+(n=75)$} & \multicolumn{2}{|c|}{$\mathrm{IR}-(n=25)$} & \\
\hline Age & $50.36 \pm 7.09$ & \multicolumn{2}{|c|}{$46.43 \pm 8.26$} & \multicolumn{2}{|c|}{$57.60 \pm 9.22$} & \multicolumn{2}{|c|}{$60.04 \pm 7.72$} & $<0.001$ \\
\hline Weight & $76.31 \pm 9.73$ & \multicolumn{2}{|c|}{$71.44 \pm 8.30$} & \multicolumn{2}{|c|}{$87.38 \pm 15.06$} & \multicolumn{2}{|c|}{$73.72 \pm 14.04$} & $<0.001$ \\
\hline Height & $167.50 \pm 7.38$ & \multicolumn{2}{|c|}{$167.48 \pm 7.87$} & \multicolumn{2}{|c|}{$162.57 \pm 9.11$} & \multicolumn{2}{|c|}{$164.16 \pm 8.60$} & 0.002 \\
\hline BMİ & $27.24 \pm 3.51$ & \multicolumn{2}{|c|}{$25.47 \pm 2.57$} & \multicolumn{2}{|c|}{$33.11 \pm 5.29$} & \multicolumn{2}{|c|}{$27.27 \pm 4.34$} & $<0.001$ \\
\hline Sex & $\%$ & $n$ & $\%$ & $n$ & $\%$ & $n$ & $\%$ & $p$ \\
\hline Female & 50.0 & 40 & 51.3 & 39 & 52.0 & 14 & 56.0 & 0.976 \\
\hline Male & 50.0 & 38 & 48.7 & 36 & 48.0 & 11 & 44.0 & \\
\hline
\end{tabular}

Table 2 Biochemical features of the cases and controls

\begin{tabular}{|c|c|c|c|c|c|c|}
\hline \multirow[t]{2}{*}{ Group } & \multicolumn{2}{|c|}{ Non-diabetic $($ mean $\pm S D)$} & \multicolumn{2}{|c|}{ Type 2 diabetic (mean \pm SD) } & \multirow[t]{2}{*}{$p$} & \multirow[t]{2}{*}{ Multiple comparison } \\
\hline & $\begin{array}{l}1 \\
\mathrm{IR}+(n=22)\end{array}$ & $\begin{array}{l}2 \\
\mathrm{IR}-(n=78)\end{array}$ & $\begin{array}{l}3 \\
\mathrm{IR}+(n=75)\end{array}$ & $\begin{array}{l}4 \\
\mathrm{IR}-(n=25)\end{array}$ & & \\
\hline Glucose (mg/dl) & $108.22 \pm 40.62$ & $92.48 \pm 28.14$ & $164.96 \pm 52.81$ & $139.84 \pm 51.18$ & $<0.001$ & $\begin{array}{l}1-3: p<0.001 \\
2-3: p<0.001 \\
2-4: p<0.001\end{array}$ \\
\hline Insulin (U/1) & $17.54 \pm 5.76$ & $7.01 \pm 3.22$ & $17.77 \pm 11.38$ & $7.31 \pm 5.62$ & $<0.001$ & $\begin{array}{l}1-2: p<0.001 \\
1-4: p<0.001 \\
2-3: p<0.001 \\
3-4: p<0.001\end{array}$ \\
\hline $\operatorname{HbA} 1 \mathrm{C}(\mathrm{mg} / \mathrm{dl})$ & $5.65 \pm 1.20$ & $5.18 \pm 0.31$ & $7.91 \pm 1.85$ & $7.24 \pm 1.99$ & $<0.001$ & $\begin{array}{l}1-3: p<0.001 \\
1-4: p=0.001 \\
2-3: p<0.001 \\
2-4: p<0.001\end{array}$ \\
\hline Triglyceride (mg/dl) & $152.09 \pm 101.58$ & $120.03 \pm 50.60$ & $183.85 \pm 94.30$ & $159.72 \pm 66.94$ & $<0.001$ & $\begin{array}{l}2-3: p<0.001 \\
2-4: p=0.042\end{array}$ \\
\hline $\mathrm{LDL}(\mathrm{mg} / \mathrm{dl})$ & $128.59 \pm 34.14$ & $133.41 \pm 44.83$ & $128.74 \pm 31.44$ & $139.20 \pm 22.81$ & 0.338 & NA \\
\hline Creatinine (mg/dl) & $0.96 \pm 0.52$ & $0.85 \pm 0.23$ & $0.90 \pm 0.29$ & $0.85 \pm 0.20$ & 0.749 & NA \\
\hline
\end{tabular}


Table 3 Genotype distributions of TLR4, IRAK1, and TIRAP in study population

\begin{tabular}{|c|c|c|c|c|c|c|c|c|c|c|}
\hline \multirow[t]{2}{*}{ Gene } & \multirow[t]{2}{*}{ Variation } & \multirow[t]{2}{*}{ Genotype } & \multicolumn{2}{|l|}{ Non-diabetic } & \multicolumn{2}{|l|}{ Type 2 DM } & \multirow[t]{2}{*}{ Statistic } & \multirow[t]{2}{*}{ Non-diabetic } & \multirow[t]{2}{*}{ Type 2 DM } & \multirow[t]{2}{*}{ Statistic } \\
\hline & & & IR (+) & $\operatorname{IR}(-)$ & IR $(+)$ & IR (-) & & & & \\
\hline \multirow[t]{8}{*}{ TLR4 } & \multirow[t]{4}{*}{ rs4986790 } & AA & $22(\% 100.0)$ & $74(\% 94.9)$ & $73(\% 97.3)$ & $23(\% 92.0)$ & \multirow[t]{4}{*}{$p=0.543$} & $96(\% 96)$ & $96(\% 96)$ & \multirow[t]{4}{*}{$p=1.000$} \\
\hline & & AG & $0(\% 0.0)$ & $4(\% 5.1)$ & $2(\% 2.7)$ & $2(\% 8.0)$ & & $4(\% 4)$ & $4(\% 4)$ & \\
\hline & & GG & $0(\% 0.0)$ & $0(\% 0.0)$ & $0(\% 0.0)$ & $0(\% 0.0)$ & & $0(\% 0.0)$ & $0(\% 0.0)$ & \\
\hline & & Total & $22(\% 100)$ & $78(\% 100)$ & $75(\% 100)$ & $25(\% 100)$ & & $100(\% 100)$ & $100(\% 100)$ & \\
\hline & \multirow[t]{4}{*}{ rs4986791 } & $\mathrm{CC}$ & $22(\% 100.0)$ & $74(\% 94.9)$ & $72(\% 96.0)$ & $23(\% 92.0)$ & \multirow[t]{4}{*}{$p=0.644$} & $96(\% 96)$ & $95(\% 95)$ & \multirow[t]{4}{*}{$p=1.000$} \\
\hline & & CT & $0(\% \quad 0.0)$ & $4(\% 5.1)$ & $3(\% 4.0)$ & $2(\% 8.0)$ & & $4(\% 4)$ & $5(\% 5)$ & \\
\hline & & $\mathrm{TT}$ & $0(\% \quad 0.0)$ & $0(\% \quad 0.0)$ & $0(\% \quad 0.0)$ & $0(\% \quad 0.0)$ & & $0(\% 0.0)$ & $0(\% 0.0)$ & \\
\hline & & Total & $22(\% 100)$ & $78(\% 100)$ & $75(\% 100)$ & $25(\% 100)$ & & $100(\% 100)$ & $100(\% 100)$ & \\
\hline \multirow[t]{12}{*}{ IRAK1 } & \multirow[t]{4}{*}{ rs 1059703} & $\mathrm{CC}$ & $2(\% 9.1)$ & $13(\% 16.7)$ & $13(\% 17.3)$ & $6(\% 24.0)$ & \multirow[t]{4}{*}{$p=0.469$} & $15(\% 15)$ & $19(\% 19)$ & \multirow[t]{4}{*}{$p=0.150$} \\
\hline & & $\mathrm{CT}$ & $3(\% 13.6)$ & $13(\% 16.7)$ & $20(\% 26.7)$ & $5(\% 20.0)$ & & $16(\% 16)$ & $25(\% 25)$ & \\
\hline & & $\mathrm{TT}$ & $17(\% 77.3)$ & $52(\% 66.7)$ & $42(\% 56.0)$ & $14(\% 56.0)$ & & $69(\% 69)$ & $56(\% 56)$ & \\
\hline & & Total & $22(\% 100)$ & $78(\% 100)$ & $75(\% 100)$ & $25(\% 100)$ & & $100(\% 100)$ & $100(\% 100)$ & \\
\hline & \multirow[t]{4}{*}{ rs3027898 } & AA & $16(\% 72.7)$ & $50(\% 64.1)$ & $41(\% 54.7)$ & $14(\% 56.0)$ & \multirow[t]{4}{*}{$p=0.544$} & $66(\% 66)$ & $55(\% 55)$ & \multirow[t]{4}{*}{$p=0.270$} \\
\hline & & $\mathrm{CA}$ & $3(\% 13.6)$ & $14(\% 17.9)$ & $20(\% 26.7)$ & $4(\% 16.0)$ & & $17(\% 17)$ & $24(\% 24)$ & \\
\hline & & $\mathrm{CC}$ & $3(\% 13.6)$ & $14(\% 17.9)$ & $14(\% 18.7)$ & $7(\% 28.0)$ & & $17(\% 17)$ & $21(\% 21)$ & \\
\hline & & Total & $22(\% 100)$ & $78(\% 100)$ & $75(\% 100)$ & $25(\% 100)$ & & $100(\% 100)$ & $100(\% 100)$ & \\
\hline & \multirow[t]{4}{*}{ rs7061789 } & AA & $16(\% 72.7)$ & $50(\% 64.1)$ & $41(\% 54.7)$ & $14(\% 56.0)$ & \multirow[t]{4}{*}{$p=0.686$} & $66(\% 66)$ & $55(\% 55)$ & \multirow[t]{4}{*}{$p=0.251$} \\
\hline & & GA & $3(\% 13.6)$ & $14(\% 17.9)$ & $20(\% 26.7)$ & $5(\% 20.0)$ & & $17(\% 17)$ & $25(\% 25)$ & \\
\hline & & GG & $3(\% 13.6)$ & $14(\% 17.9)$ & $14(\% 18.7)$ & $6(\% 24.0)$ & & $17(\% 17)$ & $20(\% 20)$ & \\
\hline & & Total & $22(\% 100)$ & $78(\% 100)$ & $75(\% 100)$ & $25(\% 100)$ & & $100(\% 100)$ & $100(\% 100)$ & \\
\hline \multirow[t]{8}{*}{ TIRAP } & \multirow[t]{4}{*}{ rs8177374 } & $\mathrm{CC}$ & $18(\% 81.8)$ & $61(\% 78.2)$ & $61(\% 81.3)$ & $23(\% 92.0)$ & \multirow[t]{4}{*}{$p=0.268$} & $79(\% 79)$ & $84(\% 84)$ & $p=0.162$ \\
\hline & & $\mathrm{CT}$ & $4(\% 18.2)$ & $17(\% 21.8)$ & $13(\% 17.3)$ & $1(\% 4.0)$ & & $21(\% 21)$ & $14(\% 14)$ & \\
\hline & & $\mathrm{TT}$ & $0(\% \quad 0.0)$ & $0(\% \quad 0.0)$ & $1(\% 1.3)$ & $1(\% 4.0)$ & & $0(\% 0.0)$ & $2(\% 2)$ & \\
\hline & & Total & $22(\% 100)$ & $78(\% 100)$ & $75(\% 100)$ & $25(\% 100)$ & & $100(\% 100)$ & $100(\% 100)$ & \\
\hline & rs8177400 & GG & $22(\% 100.0)$ & $76(\% 97.4)$ & $73(\% 97.3)$ & $24(\% 96.0)$ & $p=0.883$ & $98(\% 98)$ & $97(\% 97)$ & $p=1.000$ \\
\hline & & GA & $0(\% \quad 0.0)$ & $2(\% 2.6)$ & $1(\% 1.3)$ & $1(\% 4.0)$ & & $2(\% 2)$ & $2(\% 2)$ & \\
\hline & & AA & $0(\% \quad 0.0)$ & $0(\% \quad 0.0)$ & $1(\% 1.3)$ & $0(\% \quad 0.0)$ & & $0(\% 0.0)$ & $1(\% 1)$ & \\
\hline & & Total & $22(\% 100)$ & $78(\% 100)$ & $75(\% 100)$ & $25(\% 100)$ & & $100(\% 100)$ & $100(\% 100)$ & \\
\hline
\end{tabular}

\section{Serum IL1- $\beta$, IL6, MCP-1, and TNF- $\alpha$ levels}

When the IL1- $\beta$, IL6, MCP-1, and TNF- $\alpha$ levels of nondiabetic and T2DM individuals were compared, the levels of IL1- $\beta$, IL6, MCP-1, and TNF- $\alpha$ were significantly different in IR subgroups $(p<0.001$ and $p=0.004)$.

In the multiple comparison, T2DM subgroups showed increased IL1- $\beta$ levels compared to non-diabetic IR $(+)$ group $(p=0.018$ and $p<0.001)$ and compared to non-diabetic IR( $(-)$ group $(p=0.015$ and $p<0.001)$.

The IL6 level was significantly decreased in the T2DM subgroups compared to the non-diabetic IR(-) group $(p<0.001)$.

MCP-1 levels were significantly decreased in non-diabetic subgroups compared to T2DM subgroups $(p<0.001)$.

When TNF- $\alpha$ levels of non-diabetic IR( -$)$ group were compared to T2DM subgroups, TNF- $\alpha$ levels of IR(+) and $\operatorname{IR}(-)$ group were found to be increased ( $p=0.041$ and $p=0.031$, respectively) (Table 4 ).

\section{Evaluation of IL1- $\beta$, IL6, MCP-1, and TNF- $\alpha$ levels according to genotypes}

\section{TLR4 gene rs4986790 variant}

When the levels of IL1- $\beta$, IL6, MCP-1, and TNF- $\alpha$ were compared with genotypes of the TLR4 gene rs 4986790 variant, no significant difference was found between the levels of IL1- $\beta$, MCP-1, and TNF- $\alpha$ with genotypes $(p>0.05)$ (Table 5).

The levels of IL6 were significantly higher in individuals with AA genotypes compared to AG genotypes $(p=0.005)$ (Table 5).

\section{TLR4 gene rs4986791 variant}

When genotypes of the TLR4 gene rs4986791 were compared with IL1- $\beta$, IL6, MCP-1, and TNF- $\alpha$ levels, no 
Table 4 Serum IL1 $\beta$, IL6, MCP1, and TNF $\alpha$ levels of study population

\begin{tabular}{|c|c|c|c|c|c|c|}
\hline \multirow[t]{2}{*}{ Group } & \multicolumn{2}{|c|}{ Non-diabetic $($ mean $\pm \mathrm{SD})$} & \multicolumn{2}{|c|}{ Type 2 DM $($ mean \pm SD $)$} & \multirow[t]{2}{*}{$p$} & \multirow[t]{2}{*}{ Multiple comparison } \\
\hline & (1) $\mathrm{IR}+(n=22)$ & (2) IR- $(n=78)$ & (3) $\mathrm{IR}+(n=75)$ & (4) $\mathrm{IR}-(n=25)$ & & \\
\hline $\operatorname{IL} 1-\beta(\mathrm{pg} / \mathrm{ml})$ & $110.54 \pm 44.00$ & $106.57 \pm 63.87$ & $75.31 \pm 48.50$ & $46.28 \pm 22.97$ & $<0.001$ & $\begin{array}{l}1-3: p=0.018 \\
1-4: p<0.001 \\
2-3: p=0.015 \\
2-4: p<0.001\end{array}$ \\
\hline IL6 (pg/ml) & $79.14 \pm 49.69$ & $106.95 \pm 67.46$ & $54.02 \pm 21.16$ & $48.07 \pm 18.42$ & $<0.001$ & $\begin{array}{l}2-3: p<0.001 \\
2-4: p<0.001\end{array}$ \\
\hline MCP-1 (pg/ml) & $14.82 \pm 3.85$ & $15.57 \pm 5.46$ & $32.92 \pm 23.69$ & $26.25 \pm 8.16$ & $<0.001$ & $\begin{array}{l}1-3: p<0.001 \\
1-4: p<0.001 \\
2-3: p<0.001 \\
2-4: p<0.001\end{array}$ \\
\hline $\mathrm{TNF}-\alpha(\mathrm{pg} / \mathrm{ml})$ & $109.16 \pm 47.25$ & $110.71 \pm 57.25$ & $94.77 \pm 81.40$ & $80.93 \pm 71.87$ & 0.004 & $\begin{array}{l}2-3: p=0.041 \\
2-4: p=0.031\end{array}$ \\
\hline
\end{tabular}

Table 5 The comparison of TLR4 genotypes and IL1 $\beta$, IL6, MCP1, and TNF $\alpha$ levels

\begin{tabular}{|c|c|c|c|c|c|c|c|c|}
\hline \multicolumn{9}{|c|}{ TLR4 gene variations } \\
\hline \multirow[t]{2}{*}{ Group } & \multicolumn{4}{|l|}{ rs4986790 } & \multicolumn{4}{|l|}{ rs4986791 } \\
\hline & Genotype & $n$ & $M \pm \mathrm{SD}$ & $p$ & Genotype & $n$ & $M \pm \mathrm{SD}$ & $p$ \\
\hline \multirow[t]{3}{*}{ IL $1-\beta$} & $\mathrm{AA}$ & 192 & $89.08 \pm 56.48$ & \multirow[t]{3}{*}{0.057} & $\mathrm{CC}$ & 191 & $89.44 \pm 56.41$ & \multirow{3}{*}{$\begin{array}{l}b^{*} \\
0.020\end{array}$} \\
\hline & AG & 8 & $55.59 \pm 49.72$ & & $\mathrm{CT}$ & 9 & $51.85 \pm 47.85$ & \\
\hline & GG & 0 & - & & TT & 0 & - & \\
\hline \multirow[t]{3}{*}{ IL6 } & AA & 192 & $78.25 \pm 54.24$ & \multirow{3}{*}{$\begin{array}{l}a^{*} \\
0.005\end{array}$} & $\mathrm{CC}$ & 191 & $78.46 \pm 54.30$ & \multirow{3}{*}{$\begin{array}{l}c^{*} \\
0.003\end{array}$} \\
\hline & AG & 8 & $39.09 \pm 7.78$ & & $\mathrm{CT}$ & 9 & $39.04 \pm 7.28$ & \\
\hline & GG & 0 & - & & TT & 0 & - & \\
\hline \multirow[t]{3}{*}{ MCP-1 } & AA & 192 & $23.44 \pm 17.44$ & \multirow[t]{3}{*}{0.852} & $\mathrm{CC}$ & 191 & $23.47 \pm 17.48$ & \multirow[t]{3}{*}{0.906} \\
\hline & $\mathrm{AG}$ & 8 & $20.58 \pm 11.84$ & & $\mathrm{CT}$ & 9 & $20.23 \pm 11.13$ & \\
\hline & GG & 0 & - & & $\mathrm{TT}$ & 0 & - & \\
\hline \multirow[t]{3}{*}{ TNF- $\alpha$} & $\mathrm{AA}$ & 192 & $102.65 \pm 69.29$ & \multirow[t]{3}{*}{0.176} & $\mathrm{CC}$ & 191 & $102.96 \pm 69.33$ & \multirow[t]{3}{*}{0.129} \\
\hline & $\mathrm{AG}$ & 8 & $57.36 \pm 19.66$ & & $\mathrm{CT}$ & 9 & $55.83 \pm 18.96$ & \\
\hline & GG & 0 & - & & TT & 0 & - & \\
\hline \multirow{2}{*}{$\begin{array}{l}\text { Multiple } \\
\text { comparison }\end{array}$} & \multirow[t]{2}{*}{ IL6 } & \multirow{2}{*}{\multicolumn{2}{|c|}{$\mathrm{a}^{*} \mathrm{AA}-\mathrm{AG}: p=0.005$}} & & IL1- $\beta$ & $\mathrm{b}^{*} \mathrm{CC}-\mathrm{CT}: p=0.020$ & & \\
\hline & & & & & IL6 & $c^{*}$ CC-CT: $p=0.003$ & & \\
\hline
\end{tabular}

significant difference was found between genotypes and MCP-1 and TNF- $\alpha$ levels $(p>0.05)$.

When IL1- $\beta$ levels were compared, IL1- $\beta$ levels were found to be significantly higher in individuals with $\mathrm{CC}$ genotype than in individuals with CT genotype $(p=0.020)$.

Significantly higher IL6 levels were determined in individuals with $\mathrm{CC}$ genotype compared to individuals with CT genotypes when IL6 levels were compared $(p=0.003)$ (Table 5).

\section{IRAK1 gene rs1059703 variants}

When the levels of IL1- $\beta$, IL6, MCP-1, and TNF- $\alpha$ were compared with the genotypes of the IRAK 1 gene rs1059703 variant, no significant difference was found between the genotypes and serum IL1- $\beta$ and IL6 levels $(p>0.05)$.

MCP-1 levels were significantly different in TT, CT, and $\mathrm{CC}$ genotypes $(p=0.022)$, whereas individuals with TT genotypes had significantly lower MCP-1 levels compared to CT genotypes $(p=0.018)$.

TNF- $\alpha$ levels were found significantly different in genotype comparison ( $p=0.014$ ), whereas individuals with CT genotypes had significantly higher levels of TNF- $\alpha$ than CC genotypes $(p=0.013)$ (Table 6). 


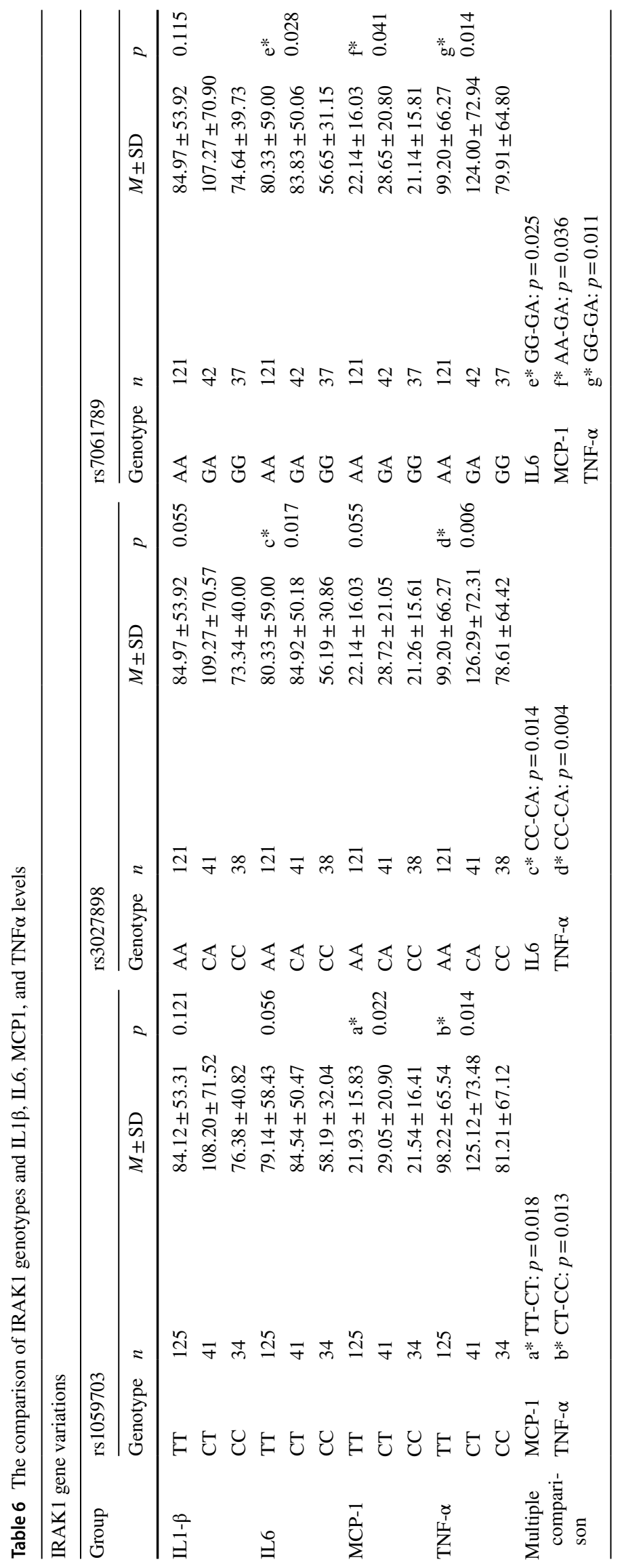




\section{IRAK1 gene rs3027898 variant}

When the levels of IL1- $\beta$, IL6, MCP-1, and TNF- $\alpha$ were compared with the genotypes of the IRAK1 gene rs3027898 variant, no difference was found between genotypes and IL1- $\beta$ and MCP-1 levels $(p>0.05)$.

There was a significant difference in IL6 levels and genotypes ( $p=0.017$ ) by multiple comparisons, and significantly lower levels of IL6 were determined in individuals with $\mathrm{CC}$ genotype compared to individuals with CA genotype $(p=0.014)$.

TNF- $\alpha$ levels were significantly different between rs3027898 genotypes $(p=0.006)$, and significantly lower levels of TNF- $\alpha$ were determined in subjects with CC genotypes compared to individuals with CA genotype $(p=0.004)$ (Table 6).

\section{IRAK1 gene rs7061789 variant}

When the levels of IL1- $\beta$, IL6, MCP-1, and TNF- $\alpha$ were compared with the genotypes of the IRAK1 gene rs7061789 variant, no difference was found between the genotypes and IL $1-\beta$ levels $(p>0.05)$.

There was a significant difference between IL6 levels and rs7061789 genotypes ( $p=0.028$ ), whereas the levels of IL6 were significantly lower in individuals with GG genotype compared to GA genotype $(p=0.025)$.

MCP-1 levels were significantly different compared to genotypes $(p=0.041)$, and individuals with AA genotypes had significantly lower MCP-1 levels than those with GA genotype $(p=0.036)$.
When TNF- $\alpha$ levels were compared, there was a significant difference in AA, GA, and GG genotypes $(p=0.014)$, significantly lower levels of TNF- $\alpha$ were determined in subjects with GG genotype compared to individuals with GA genotype $(p=0.011)$ (Table 6).

\section{TIRAP gene rs8177374 variant}

When the genotypes of TIRAP gene rs 8177374 were compared with IL1- $\beta$, IL6, MCP-1, and TNF- $\alpha$ levels, no significant difference was found between genotypes and IL1- $\beta$ and MCP-1 levels $(p>0.05)$.

There was a significant difference in CC, CT and TT genotypes when IL6 levels were compared $(p=0.001)$, and significantly lower IL6 levels were determined in individuals with CC genotypes compared to individuals with CT genotype $(p=0.001)$.

When TNF- $\alpha$ levels were compared, there was a significant difference in CC, CT, and TT genotypes $(p=0.020)$, whereas significantly lower levels of TNF- $\alpha$ were determined in individuals with $\mathrm{CC}$ genotype compared to individuals with CT genotype $(p=0.030)$ (Table 7).

\section{TIRAP gene rs 8177400 variant}

When genotypes of TIRAP gene rs 8177400 were compared with IL1- $\beta$, IL6, MCP-1, and TNF- $\alpha$ levels, no significant difference was found between genotypes and IL1- $\beta$, IL6, MCP-1, and TNF- $\alpha$ levels ( $p>0.05)$ (Table 7).

Table 7 The comparison of TIRAP genotypes and IL1 $\beta$, IL6, MCP1, and TNF $\alpha$ levels

\begin{tabular}{|c|c|c|c|c|c|c|c|c|}
\hline \multicolumn{9}{|c|}{ TIRAP gene variations } \\
\hline \multirow[t]{2}{*}{ Group } & \multicolumn{4}{|l|}{ rs 8177374} & \multicolumn{4}{|l|}{ rs8177400 } \\
\hline & Genotype & $n$ & $M \pm \mathrm{SD}$ & $p$ & Genotype & $n$ & $M \pm \mathrm{SD}$ & $p$ \\
\hline \multirow[t]{3}{*}{ IL1- $\beta$} & $\mathrm{CC}$ & 163 & $85.73 \pm 57.20$ & \multirow[t]{3}{*}{0.380} & GG & 195 & $87.71 \pm 55.02$ & \multirow[t]{3}{*}{0.253} \\
\hline & $\mathrm{CT}$ & 35 & $96.21 \pm 53.67$ & & GA & 4 & $84.30 \pm 125.80$ & \\
\hline & TT & 2 & $103.70 \pm 59.11$ & & AA & 1 & 107.25 & \\
\hline \multirow[t]{3}{*}{ IL6 } & $\mathrm{CC}$ & 163 & $72.41 \pm 53.48$ & \multirow{3}{*}{$\begin{array}{l}\mathrm{a}^{*} \\
0.001\end{array}$} & GG & 195 & $77.22 \pm 53.98$ & \multirow[t]{3}{*}{0.235} \\
\hline & $\mathrm{CT}$ & 35 & $97.76 \pm 51.57$ & & GA & 4 & $57.01 \pm 46.22$ & \\
\hline & TT & 2 & $56.42 \pm 30.70$ & & $\mathrm{AA}$ & 1 & 49.93 & \\
\hline \multirow[t]{3}{*}{ MCP-1 } & $\mathrm{CC}$ & 163 & $22.73 \pm 15.70$ & \multirow[t]{3}{*}{0.152} & GG & 195 & $23.25 \pm 17.29$ & \multirow[t]{3}{*}{0.330} \\
\hline & CT & 35 & $24.16 \pm 20.89$ & & GA & 4 & $19.66 \pm 6.69$ & \\
\hline & TT & 2 & $57.05 \pm 44.67$ & & AA & 1 & 53.52 & \\
\hline \multirow[t]{3}{*}{ TNF- $\alpha$} & $\mathrm{CC}$ & 163 & $95.91 \pm 65.80$ & \multirow{3}{*}{$\begin{array}{l}\mathrm{b}^{*} \\
0.020\end{array}$} & GG & 195 & $100.92 \pm 68.06$ & \multirow[t]{3}{*}{0.121} \\
\hline & $\mathrm{CT}$ & 35 & $127.83 \pm 75.64$ & & GA & 4 & $68.28 \pm 80.33$ & \\
\hline & TT & 2 & $30.46 \pm 1.73$ & & AA & 1 & 214.85 & \\
\hline \multirow{2}{*}{$\begin{array}{l}\text { Multiple com- } \\
\text { parison }\end{array}$} & IL6 & $\mathrm{a}^{*} \mathrm{CC}-\mathrm{CT}: p=0.001$ & & & & & & \\
\hline & TNF- $\alpha$ & $\mathrm{b}^{*} \mathrm{CC}-\mathrm{CT}: p=0.030$ & & & & & & \\
\hline
\end{tabular}




\section{Discussion}

Type 2 diabetes is a complex and chronic disease caused by a combination of insulin secretion and insulin resistance from pancreatic beta cells. According to the hypothesis of meta-inflammation (metabolically induced inflammation), preclinical chronic low-grade inflammation due to changes in the immune response, which is the first line of defense against viruses, bacteria and fungi, is similarly occurring in the case of type 2 diabetes and insulin resistance [20].

At this point, the natural immune response against many pathogens provided with the transmembrane protein family named Toll-like receptors. Inflammation disrupts insulin sensitivity by activation of the Toll-like receptor family, particularly TLR4. Therefore, polymorphisms in geneencoding proteins in the natural immune system, such as Toll-like receptors, may affect the immune response and T2DM development [5, 6]. Activation of TLR4 with LPS results in NF- $\kappa B$ activation, and thus, the expression of inflammatory regulatory genes such as TNF- $\alpha$, IL- 1 , IL-6, and MCP-1. Activation of the proinflammatory pathway also results in insulin resistance and type 2 diabetes.

Our study was planned to investigate the TLR4 and TLR4 inflammation pathways, which are closely related to type 2 diabetes and insulin resistance, and that a functional polymorphism that could occur in any of the members of the pathway would impair the functioning of the pathway and cause damage in the mechanism of inflammation.

In our study, TLR4 (rs4986790, rs4986791), IRAK1 (rs1059703, rs3027898, rs7061789), and TIRAP (rs8177374, rs8177400) genes on a total of 7 with SNP variation, and serum levels of TNF- $\alpha$, IL-6, MCP-1, and IL- $1 \beta$ were investigated. The results of our findings are discussed below.

\section{Gene variants}

\section{IRAK1 gene rs1059703, rs3027898, and rs7061789 variants}

IRAK 1 gene is $8.4 \mathrm{kbp}$ long and is located in the pseudoautosomal (PAR2) region of the $\mathrm{X}$ chromosome (Xq28) [21].

Lakoski et al. reported that the haplotypes of the IRAK1 variants rs3027898 and rs 7061789 were associated with C-reactive protein levels [21].

Yoshida et al. found that IRAK1Ser532Leu (rs 1059703) polymorphism was associated with chronic kidney disease in type 2 diabetic patients [22].

Similarly, the polymorphisms identified in the IRAK1 gene indicated that the $\mathrm{T}$ allele in $1595 \mathrm{C} / \mathrm{T}$ (rs1059703) had a protective effect against chronic kidney disease in type 2 diabetes and had vascular inflammation-relieving effect. In addition, the $\mathrm{C}$ allele at rs 1059703 and rs3027898 has been reported to be associated with increased NF-kB activation [23].

Although there is no study investigating the association of IRAK1 gene rs1059703, rs3027898, and rs7061789 variants with diabetes risk, our study was not associated with these variants and type 2 diabetes.

\section{TLR4 gene rs4986790 and rs4986791 variants}

The TLR 4 gene consists of 3 exons and is localized to chromosome 9q32-33. In the exon 3 of the human TLR4 gene, two single-nucleotide polymorphisms (SNPs) have been widely described. These are 896 A/G (rs4986790) and 1196 $\mathrm{C} / \mathrm{T}$ (rs4986791) and including amino acid modification at position 299 and 399, respectively [16].

These two polymorphisms in the TLR4 gene have been reported to be responsible for the reduction of response to LPS. Their association with T2DM and IR+ has been investigated in some genetic studies, but the disease is not fully associated with TLR4 variations. Unlike these studies, some studies show that TLR4 rs4986790 and rs4986791 polymorphisms are associated with T2DM [20].

Kolz et al. reported that minor alleles in TLR4 variants were not directly related to type 2 diabetes, but increased the risk of type 2 diabetes with high cholesterol levels [24].

Bagarolli et al. reported that TLR4 Asp299Gly and Thr399Ile variants had an effect on the development of type 2 diabetes and similar results were obtained in some chronic diseases such as coronary artery disease [25].

In another study conducted in patients with diabetic retinopathy, Asp299Gly and Thr399Ile variants of the TLR 4 gene also showed heterozygous distribution in patients with diabetic retinopathy [16].

Mesci et al. found no association between TLR4 Asp299Gly and Thr399Ile and type 2 diabetes in the Turkish population [26].

Similarly, we found no association between the TLR4 gene rs 4986790 and rs 4986791 variants with type 2 diabetes and insulin resistance.

\section{TIRAP gene rs8177374 and rs8177400 variants}

TIRAP gene is $14,500 \mathrm{bp}$ long, localized at chromosome 11 (11q24.2) and encodes $25 \mathrm{kDa}$ proteins containing 221 amino acids. Three different polymorphic regions were widely studied for the TIRAP gene. These are C558T (rs7932766), C539T (rs8177374), and G286A (rs8177400) [27].

It was reported that the $11631 \mathrm{G} / \mathrm{A}$ (rs8177400) variant caused loss of function of the TIRAP/MAL protein as a 
result of which the Mal-MyD88 interaction was impaired or the Mal-MyD88 complex was transported to the plasma membrane and NF-kB activation and IL-6, IL-8, and TNF has been reported to be necessary for production [23].

Liu et al. found a link between TIRAP C539T polymorphism and low risk of tuberculosis infection, and showed that this polymorphism was protective against tuberculosis [27].

In another study, the association of TIRAP rs 8177400 variant with lymphoma risk was investigated, and no correlation was found between this variant and lymphoma risk [28].

There is no study investigating the relationship between TIRAP gene rs 8177374 and rs8177400 variants with diabetes risk and, no correlation was found between these variants and the risk of type 2 diabetes in our study.

\section{Inflammatory factors}

Most of the proinflammatory adipokines such as IL- $1 \beta$, IL-6, IL- 8 , TNF- $\alpha$, and MCP-1 are expressed by white adipose tissue. Activation of TLR4 with long-chain fatty acids and LPS activates MyD88-dependent pathway, resulting in NF-kB activation and, thus, expression of inflammatory regulatory genes such as TNF- $\alpha$, IL-1, IL-6, and MCP-1 [11].

In our study, IL1- $\beta$, IL6, MCP-1, and TNF- $\alpha$ levels were measured in IR+/IR- subgroups of non-diabetic and T2DM subjects, and there was a significant difference between IL1$\beta$, IL6, MCP-1, and TNF- $\alpha$ levels. In this section, the findings of non-diabetic and T2DM individuals on inflammatory mediator levels will be discussed.

\section{IL1- $\beta$ levels}

$\mathrm{IL}-1 \beta$ is an important inflammatory regulator and plays an important role in the pathophysiology of many diseases such as type 1 diabetes, type 2 diabetes, and metabolic syndrome [29].

IL- $1 \alpha$ and IL-1 $\beta$ are among the first identified cytokines and have strong proinflammatory functions. Interleukindeficient mice have lower fasting glucose and insulin levels and higher insulin sensitivity. IL-1 $\beta$ and IL- 6 measurements are necessary for effective T2DM diagnosis. IL-1 $\beta$ is also associated with insulin receptor substrate-1 expression [8].

Persaud et al. reported that IL1- $\beta$ levels increased in mouse $\beta$-cells due to hyperglycemia similar to COX-2 levels and IL1- $\beta$ levels were associated with $\beta$-cell dysfunction [30].

In a study by Maedler et al., they reported that there was no IL1- $\beta$-producing $\beta$-cell structure in pancreatic sections of non-diabetic individuals in vivo, which is present in T2DM patients [31].
Similarly, in our study, IL1- $\beta$ levels were increased in T2DM IR+ and IR- group compared to non-diabetic IR+/ IR- group. Consistent with the literature, high levels of IL1- $\beta$ in T2DM individuals are thought to be associated with hyperglycemia.

\section{IL6 levels}

IL6 is a protein of about $26 \mathrm{kDa}$ which is one of the first cytokines associated with IR. IL6 is synthesized from mononuclear phagocytes, vascular endothelial cells, fibroblasts, and some activated T cells with epithelial cells. IL6 and TNF- $\alpha$ have been found to act on insulin resistance and type 2 diabetes by competing with insulin signaling pathways. In patients, the IL6 concentration decreases parallel to weight loss and IR development. The production of IL6 in the abdominal tissue is at least threefold higher than the subcutaneous adipose tissue and, thus, contributing partly to the hepatic IR. IL6 increases fat oxidation in humans in vivo [8].

Pickup et al. reported increased levels of IL6 in patients with T2DM and features of insulin resistance syndrome [32].

Similarly, Kern et al. found that IL6 levels were high in individuals with low insulin sensitivity and were associated with unesterified fatty acids [33].

Carey et al. reported in their study in 2004 that IL6 levels did not increase in T2DM patients and IL6 levels were associated with fat mass and insulin insensitivity [34].

In contrast to these studies, IL6 levels were significantly lower in T2DM subgroups compared to non-diabetic IRgroup. Similar levels of triglyceride in the same groups were also different, and IL6 was associated with fatty acids, suggesting that unexpected levels of IL6 may be related to triglyceride levels.

Similarly, Khovidhunkit et al. reported that IL-6 reduced total cholesterol and triglyceride levels within $24 \mathrm{~h}$ of administration to healthy volunteers. Although the mechanism of this effect is not known exactly, IL-6 has been shown to increase hepatic fatty acid synthesis and lipolysis in adipose tissue and to decrease cholesterol secretion despite increasing cholesterol synthesis [35].

In contrast to these studies, IL6 levels were significantly lower in T2DM subgroups compared to non-diabetic IRgroup. Similar levels of triglyceride in the same groups were also different, and IL6 was associated with fatty acids, suggesting that unexpected levels of IL6 may be related to triglyceride levels.

Already, Khovidhunkit et al. reported that administration of IL-6 to healthy volunteers reduced total cholesterol and triglyceride levels within $24 \mathrm{~h}$. Although the mechanism of this effect is not known exactly, IL-6 has been shown to increase hepatic fatty acid synthesis and lipolysis in adipose tissue and to decrease cholesterol secretion despite 
increasing cholesterol synthesis [35]. Our findings are consistent with these data.

\section{MCP-1 levels}

Monocyte chemoattractant protein (MCP)-1 is a member of the chemokine receptor family and is secreted by vascular endothelial cells and macrophages in atherosclerotic lesions. MCP-1 has been reported to play a direct role in the development of insulin resistance in adipose tissue by reducing insulin receptor tyrosine phosphorylation [36]. Recent studies have reported that MCP-1 plays an important role in obesity, diabetes, cardiovascular disease, diabetic nephropathy, and retinopathy [37].

Sartipy et al. reported that MCP-1 is a protein responsible for insulin activity, that increased levels of MCP-1 induce de-differentiation (return to the undifferentiated state of cells) in adipocytes and, thus, contribute to pathologies such as obesity and hyperinsulinemia including T2DM [38].

Similarly, circulating MCP-1 levels were found to be associated with the risk of T2DM [37], and MCP-1 levels in T2DM have been reported to be higher compared to control in both rodent model and patients [39]. Therefore, the high MCP-1 levels in T2DM patients in our study were consistent with the literature.

\section{TNF-a levels}

Some mediators, which are proinflammatory cytokines released from various cells such as immune cells (monocytes, adipocytes, etc.) have been associated with IR development. One of them, TNF- $\alpha$, has an increased level of blood in patients with insulin resistance and obesity, and this increase is associated with an increase in CRP and IL-6. The main effect of TNF- $\alpha$ is the formation of a post-receptor defect at the insulin receptor [40, 41].

In a study investigating the relationship between obesity and TNF- $\alpha$, inflammation and adipocytes in obese animals have been shown that increase of TNF- $\alpha$ expression can alter insulin regulation [8].

Uysal et al. reported that insulin sensitivity increased in TNF- $\alpha$ or TNF- $\alpha$ receptor knockout mice. It has been reported that, in cells exposed to TNF- $\alpha$ and high free fatty acids at the molecular level, serine residues stimulated inhibitor phosphorylation [42].

TNF- $\alpha$ and IL- 6 have been reported to have a competitive effect with insulin signaling pathways and cause insulin resistance in patients with type 2 diabetes. There are studies showing that TNF- $\alpha$ is also associated with insulin resistance in pregnancy $[8,43]$.

Dandona et al. showed that TNF- $\alpha$ increased in pregnant women with gestational diabetes and this increase was associated with oxidative stress and inflammatory changes caused by hyperglycemia. It is thought that TNF- $\alpha$ elevates intracellular receptor autophosphorylation and IRS phosphorylation, causing post-receptor defects and, thus, prevents insulin from acting on target tissue $[8,43]$.

It has been thought that TNF- $\alpha$ and IL- 6 cause diabetes over insulin resistance, and additionally end products of nonenzymatic glycosylation in diabetes stimulate these cytokines by binding to macrophages. Therefore, it is not yet clear whether diabetes triggers inflammation or causes inflammation or diabetes [44].

In our study, TNF- $\alpha$ levels were decreased in T2DM IR+ and IR- group compared to non-diabetic IR-group. This effect, other than the expected, may be associated with IL6 levels. Because TNF- $\alpha$ and IL-6 levels are interrelated, triglyceride levels or cytokines may affect each other's levels.

\section{The relationship of IL1- $\beta$, IL6, MCP-1, and TNF- $\alpha$ levels with gene variants}

Although the effect of genotyping results on IL1- $\beta$, IL6, MCP-1, and TNF- $\alpha$ levels will be discussed in this chapter, there is no study in the literature that evaluates these protein levels with the gene variants that we investigated except TLR4. Therefore, the interpretation of the meaningful results obtained from our findings will be discussed in this section.

\section{IRAK1 genotypes and IL6, MCP-1, and TNF-a levels}

Within the scope of our study, the genotyped IRAK1 gene was remarkable between the genotype distribution of the rs1059703, rs3027898, and rs7061789 variants, and especially the TNF- $\alpha$ levels. For these three variants of the IRAK1 gene, individuals with the heterozygous genotype were found to have increased levels of TNF- $\alpha$. Similarly, levels of MCP-1 were significantly increased for rs3027898 and rs7061789, whereas IL6 levels were significantly increased for heterozygote genotypes. These results showed that the IRAK1 gene was associated with IL6, MCP-1, and TNF- $\alpha$ levels.

\section{TLR4 genotypes and IL-1 $\beta$, IL6 levels}

When the levels of IL-1 $\beta$ and IL6 were compared with the TLR4 gene rs4986790 and rs4986791 variants, there were relationship between IL6 levels and rs4986790 variant, and IL6 and IL-1 $\beta$ levels with rs4986791 variant.

Gurung et al. found that TLR4 and IL- $1 \beta$ levels were closely related and TLR4 knockout macrophages did not develop IL-1 $\beta$ response after infection [45].

Böttcher et al. reported that TLR4 variants were associated with LPS-induced IL12 levels and that rs4986790 was associated with decreased IL12 levels [46]. 
In our study, IL6 levels for both variants were higher in individuals with ancestral genotype. For both variants, a mutant individual was not identified, but low IL6 levels in heterozygous individuals showed that mutant alleles could affect IL6 levels. The level of IL-1 $\beta$ for rs4986791 variant was found to be high in the ancestral genotype and low in the heterozygote genotype in a similar to IL6. For this variation, it can be thought that the mutant allele can be effective in decreasing the level of IL- $1 \beta$.

\section{TIRAP genotypes and IL6 and TNF-a levels}

When the protein levels of the TIRAP gene rs8177374 and rs8177400 variants were compared, it was found that there was a correlation between the genotypes of rs8177374 variant and IL6 and TNF- $\alpha$ levels.

Both proteins were significantly higher in the heterozygous genotype for the TIRAP gene rs8177374 variant. These findings showed that the mutant allele affected IL6 and TNF- $\alpha$ levels, and the TIRAP gene was associated with IL6 and TNF- $\alpha$.

\section{Conclusion}

As a result of our study, a total of seven variants of TLR4, IRAK1, and TIRAP genes were genotyped, but no correlation was found between these variants and the risk of type 2 diabetes and insulin resistance. The important point of our study is that five of these seven variations were first time genotyped in our study in patients with type 2 diabetes and insulin resistance.

TNF- $\alpha$, IL-6, MCP-1, and IL-1 $\beta$ levels were also associated with diabetes and insulin resistance. Although the gene variants were not significant in type 2 diabetes and insulin resistance groups, IRAK1, TLR4, and TIRAP gene variants were found to be associated with TNF- $\alpha$, IL-6, MCP-1, and IL-1 $\beta$ levels.

Our study is the first study to link IRAK1, TLR4, and TIRAP variants with TNF- $\alpha$, IL-6, MCP- 1 , and IL- $1 \beta$ levels according to current literature. These results are particularly important for TLR4 inflammation pathway.

Acknowledgements This study supported by the Scientific and Technological Research Council of Turkey (TUBITAK) (Project number: 113S427).

\section{References}

1. Dandona P, Aljada A, Bandyopadhyay A. Inflammation: the link between insulin resistance, obesity and diabetes. Trends Immunol. 2004;25:4-7.
2. Esser N, Legrand-Poels S, Piette J, Scheen AJ, Paquot N. Inflammation as a link between obesity, metabolic syndrome and type 2 diabetes. Diabetes Res Clin Pract. 2014;105:141-50.

3. Rogero M, Calder P. Obesity, inflammation, toll-like receptor 4 and fatty acids. Nutrients. 2018;10:432.

4. Association AD. Diagnosis and classification of diabetes mellitus. Diabetes Care. 2010;33:S62-9.

5. Moreira AB, Alfenas RDCG. The influence of endotoxemia on the molecular mechanisms of insulin resistance. Nutr Hosp. 2012;27:382-90.

6. Burtis CA, Ashwood ER, Bruns DE: Tietz textbook of clinical chemistry and molecular diagnosis. 5th ed. Elsevier. 2001. ISBN: 978-1-4160-6164-9.

7. Ozbayer C, Kebapci MN, Degirmenci I, Yagci E, Gunes HV, Kurt $H$. Genetic variant in the $3^{\prime}$-untranslated region of the COX2 gene is associated with type 2 diabetes: a hospital-based case-control study. Prostaglandins Leukot Essent Fatty Acids. 2018;137:39-42.

8. Tilg H, Moschen AR. Inflammatory mechanisms in the regulation of insulin resistance. Mol Med. 2008;14:222.

9. Shi X, Wang S, Luan H, Tuerhong D, Lin Y, Liang J, et al. Clinopodium chinense attenuates palmitic acid-induced vascular endothelial inflammation and insulin resistance through TLR4-mediated NF- $\mathrm{B}$ B and MAPK pathways. Am J Chin Med. 2019;47:97-117.

10. Ozbayer C, Kebapçı MN, Yağcı E, Kurt H, Güneş HV, Değirmenci I. The rs4925648, rs121908149 and rs121908152 genetic variations in the NLRP3 inflammasome and risk of type 2 diabetes. Osmangazi J Med. 2019;41:153-60.

11. Kanczkowski W, Ziegler C, Zacharowski K, Bornstein S. Toll-like receptors in endocrine disease and diabetes. Neuroimmunomodulation. 2008;15:54-60.

12. Kim MJ, Yoo SS, Choi Y-Y, Park JY. A functional polymorphism in the pre-microRNA-196a2 and the risk of lung cancer in a Korean population. Lung Cancer. 2010;69:127-9.

13. Zhang X-Y, Liu Y, He T, Yang T-T, Wu J, Cianflone K, et al. Anaphylatoxin $\mathrm{C} 5 \mathrm{a}$ induces inflammation and reduces insulin sensitivity by activating TLR4/NF-kB/PI3 K signaling pathway in 3T3-L1 adipocytes. Biomed Pharmacother. 2018;103:955-64.

14. Ve T, Gay NJ, Mansell A, Kobe B, Kellie S. Adaptors in toll-like receptor signaling and their potential as therapeutic targets. Curr Drug Targets. 2012;13:1360-74.

15. Browne EP. Regulation of B-cell responses by Toll-like receptors Immunology. 2012;136:370-9.

16. Buraczynska M, Baranowicz-Gaszczyk I, Tarach J, Ksiazek A. Toll-like receptor 4 gene polymorphism and early onset of diabetic retinopathy in patients with type 2 diabetes. Hum Immunol. 2009; 70:121-4.

17. Özbayer C, Kurt H, Yangı B. TLR4 ve TLR4 Sinyal Yolağındaki Genetik Varyantların İnsülin Direnci ve Diyabet Riski İle İlişkisi. J Clin Anal Med. 2014;5:168-72.

18. Gottipati S, Rao NL, Fung-Leung W-P. IRAK1: a critical signaling mediator of innate immunity. Cell Signal. 2008;20:269-76.

19. Castiblanco J, Varela D-C, Castaño-Rodríguez N, Rojas-Villarraga A, Hincapié M-E, Anaya J-M. TIRAP (MAL) S180L polymorphism is a common protective factor against developing tuberculosis and systemic lupus erythematosus. Infect Genet Evol. 2008;8:541-4.

20. Assmann T, Lemos N, de Almeida Brondani L, Carlessi R, Maldonado-Bernal C. Association between Asp299Gly and Thr399Ile polymorphisms in toll-like receptor 4 gene and type 2 diabetes mellitus: case-control study and meta-analysis. J Diabetes Metab. 2014;5:427. https://doi.org/10.4172/2155-6156.100042.

21. Lakoski SG, Li L, Langefeld CD, Liu Y, Howard TD, Brosnihan $\mathrm{KB}$, et al. The association between innate immunity gene (IRAK1) and C-reactive protein in the Diabetes Heart Study. Exp Mol Pathol. 2007;82:280-3. 
22. Yoshida T, Kato K, Yokoi K, Watanabe S, Metoki N, Yoshida H, et al. Association of genetic variants with chronic kidney disease in Japanese individuals with type 2 diabetes mellitus. Int J Mol Med. 2009;23:529-37.

23. Kutikhin AG. Association of polymorphisms in TLR genes and in genes of the Toll-like receptor signaling pathway with cancer risk. Hum Immunol. 2011;72:1095-116.

24. Kolz M, Baumert J, Müller M, Khuseyinova N, Klopp N, Thorand $\mathrm{B}$, et al. Association between variations in the TLR4 gene and incident type 2 diabetes is modified by the ratio of total cholesterol to HDL-cholesterol. BMC Med Genet. 2008;9:9.

25. Bagarolli RA, Saad MJA, Saad STO. Toll-like receptor 4 and inducible nitric oxide synthase gene polymorphisms are associated with type 2 diabetes. J Diabetes Complicat. 2010;24:192-8.

26. Mesci B, Eren P, Kiziltas S, Oguz A, Tuncer I, Kostek O, et al. Association between toll-like receptor 4 polymorphism and type 2 diabetes mellitus. Endocr Abs. 2013;32:P372. https://doi. org/10.1530/endoabs.32.P372.

27. Liu Q, Li W, Li D, Feng Y, Tao C. TIRAP C539T polymorphism contributes to tuberculosis susceptibility: evidence from a metaanalysis. Infect Genet Evol. 2014;27:32-9.

28. George J, Kubarenko AV, Rautanen A, Mills TC, Colak E, Kempf $\mathrm{T}$, et al. MyD88 adaptor-like D96N is a naturally occurring lossof-function variant of TIRAP. J Immunol. 2010;184:3025-32.

29. Dinarello CA, Donath MY, Mandrup-Poulsen T. Role of IL-1 $\beta$ in type 2 diabetes. Curr Opin Endocrinol Diabetes Obes. 2010;17:314-21.

30. Persaud SJ, Burns CJ, Belin VD, Jones PM. Glucose-induced regulation of COX-2 expression in human islets of Langerhans. Diabetes. 2004;53:S190-2.

31. Maedler K, Sergeev P, Ris F, Oberholzer J, Joller-Jemelka HI, Spinas GA, et al. Glucose-induced $\beta$ cell production of IL-1 $\beta$ contributes to glucotoxicity in human pancreatic islets. J Clin Investig. 2002;110:851-60.

32. Pickup JC, Chusney GD, Thomas SM, Burt D. Plasma interleukin-6, tumour necrosis factor $\alpha$ and blood cytokine production in type 2 diabetes. Life Sci. 2000;67:291-300.

33. Kern PA, Ranganathan S, Li C, Wood L, Ranganathan G. Adipose tissue tumor necrosis factor and interleukin- 6 expression in human obesity and insulin resistance. Am J Physiol Endocrinol Metab. 2001;280:E745-51

34. Carey A, Bruce C, Sacchetti M, Anderson M, Olsen D, Saltin B, et al. Interleukin- 6 and tumor necrosis factor- $\alpha$ are not increased in patients with type 2 diabetes: evidence that plasma interleukin-6 is related to fat mass and not insulin responsiveness. Diabetologia. 2004;47:1029-37.

35. Khovidhunkit W, Kim M-S, Memon RA, Shigenaga JK, Moser $\mathrm{AH}$, Feingold KR, et al. Effects of infection and inflammation on lipid and lipoprotein metabolism: mechanisms and consequences to the host. J Lipid Res. 2004;45:1169-96.

36. Takebayashi K, Matsumoto S, Aso Y, Inukai T. Association between circulating monocyte chemoattractant protein-1 and urinary albumin excretion in nonobese type 2 diabetic patients. J Diabetes Complicat. 2006;20:98-104.

37. Panee J. Monocyte chemoattractant protein 1 (MCP-1) in obesity and diabetes. Cytokine. 2012;60:1-12.

38. Sartipy P, Loskutoff DJ. Monocyte chemoattractant protein 1 in obesity and insulin resistance. Proc Natl Acad Sci. 2003; 100:7265-70.

39. Nomura S, Shouzu A, Omoto S, Nishikawa M, Fukuhara S. Significance of chemokines and activated platelets in patients with diabetes. Clin Exp Immunol. 2000;121:437-43.

40. Coban E, Sari R, Ozdogan M, Akcit F. Levels of plasma fibrinogen and d-dimer in patients with impaired fasting glucose. Exp Clin Endocrinol Diabetes. 2005;113:35-7.

41. Coppack SW. Pro-inflammatory cytokines and adipose tissue. Proc Nutr Soc. 2001;60:349-56.

42. Uysal KT, Wiesbrock SM, Marino MW, Hotamisligil GS. Protection from obesity-induced insulin resistance in mice lacking TNF- $\alpha$ function. Nature. 1997;389:610-4.

43. Ategbo J-M, Grissa O, Yessoufou A, Hichami A, Dramane K, Moutairou K, et al. Modulation of adipokines and cytokines in gestational diabetes and macrosomia. J Clin Endocrinol Metab. 2006;91:4137-43.

44. Yenicesu M, Yilmaz M, Caglar K, Sonmez A, Eyileten T, Kir T, et al. Adiponectin level is reduced and inversely correlated with the degree of proteinuria in type 2 diabetic patients. Clin Nephrol. 2005;64:12-9.

45. Gurung P, Malireddi RS, Anand PK, Demon D, Walle LV, Liu Z, et al. Toll or interleukin-1 receptor (TIR) domain-containing adaptor inducing interferon- $\beta$ (TRIF)-mediated caspase- 11 protease production integrates Toll-like receptor 4 (TLR4) protein-and Nlrp3 inflammasome-mediated host defense against enteropathogens. J Biol Chem. 2012;287:34474-83.

46. Böttcher MF, Hmani-Aifa M, Lindström A, Jenmalm MC, Mai $\mathrm{X}-\mathrm{M}$, Nilsson L, et al. A TLR4 polymorphism is associated with asthma and reduced lipopolysaccharide-induced interleukin-12 (p70) responses in Swedish children. J Allergy Clin Immunol. 2004;114:561-7.

Publisher's Note Springer Nature remains neutral with regard to jurisdictional claims in published maps and institutional affiliations. 